\title{
Analysis of programmed cell death in associative gene Network of glaucoma reconstructed using ANDSystem
}

\author{
O.V. Saik ${ }^{1 *}$, P.S. Demenkov ${ }^{1}$, O.S. Konovalova², N.A. Konovalova ${ }^{3}$ I.N. Lavrik ${ }^{1,4}$, \\ V.A. Ivanisenko ${ }^{1}$ \\ ${ }^{1}$ Institute of Cytology and Genetics SB RAS, Novosibirsk, Russia \\ ${ }^{2}$ Federal State Budgetary Educational Institution of Higher Education "Tyumen State Medical University" \\ of the Ministry of Healthcare of the Russian Federation, the Ekaterinburg branch of MNTK \\ Eye Microsurgery, Tyumen, Russia \\ ${ }^{3}$ Federal State Budgetary Educational Institution of Higher Education "Tyumen State Medical \\ University” of the Ministry of Healthcare of the Russian Federation, Tyumen, Russia \\ ${ }^{4}$ Otto von Guericke University Magdeburg, Magdeburg, Germany \\ *e-mail:saik@bionet.nsc.ru
}

Key words: primary open-angle glaucoma (POAG), apoptosis, ANDSystem, gene networks

Motivation and Aim: Nowadays one of the leading causes of irreversible blindness is primary open angle glaucoma (POAG) affecting more than 44 million people worldwide. Excess apoptosis is one of the factors involved in degradation of retinal ganglion cells during POAG [1]. In this work analysis of the role of apoptosis in associative gene network of POAG was performed.

Methods and Algorithms: The list of genes associated with POAG was created based on the information from databases (OMIM, ClinVar, GWAS catalog and SNPedia) and ANDSystem [2]. Genes involved in apoptosis were taken from KEGG database. Gene network reconstruction and analysis was performed using ANDSystem.

Results: Automatic analysis of information from databases and ANDSystem revealed 156 genes associated with POAG. Gene Ontology enrichment analysis showed that these genes are involved in apoptosis, extracellular matrix organization, angiogenesis and other biological processes. According to the value of betweenness centrality TP53 is one of the most central participants of both POAG and apoptosis gene networks. It was shown, for example, that SNPs in TP53 are associated with POAG and protein p53 can decrease the level of MMP1 (its level is increased in POAG). The elevated MMP1 can participate in activation of collagen degradation. Collagen breakdown, in turn, is involved in trabecular meshwork disruption, which is a known pathogenesis factor of POAG.

Conclusion: It was shown that the apoptosis pathway is tightly related to POAG gene network. Apoptosis genes play the central role in the gene network of POAG. Gene TP53 was found to be one of the most central players in both POAG gene network and apoptosis pathway.

Acknowledgements: Supported by the Russian Science Foundation grant "Programmed cell death induced via death receptors: Delineating molecular mechanisms of apoptosis initiation via molecular modeling" 14-44-00011.

\section{References}

1. Lindner E. et al. (2015) Analysis of functional polymorphisms in apoptosis-related genes in primary open angle glaucoma. Molecular Vision. 21:1340.

2. Ivanisenko V.A. et al. (2015) ANDSystem: an Associative Network Discovery System for automated literature mining in the field of biology. BMC Syst Biol. 9(Suppl 2):S2. 